\title{
ANÁLISIS DE LA EMERGENCIA DE PLÁNTULAS DURANTE LA VIVERIZACIÓN DE UNA COLECCIÓN DE SEMILLAS DE 418 FAMILIAS DE Araucaria araucana
}

Gutiérrez, Braulio²

\section{RESUMEN}

Se evalúa la emergencia de plántulas durante la viverización operacional de semillas de 418 familias de Araucaria araucana, clasificadas en 5 estratos ecológico-genéticos y caracterizadas en función de la incidencia de la enfermedad "daño foliar de la araucaria" en las madres desde donde se obtuvo las semillas.

Se observa que la emergencia de plántulas de las familias andinas es más lenta, pero alcanza valores mayores que en las familias costeras ( 81,4 y $77,9 \%$, respectivamente).

Tanto en los distintos estratos por separado, como a nivel conjunto, la emergencia de plántulas exhibe relación directa con el tamaño de las semillas.

Respecto a las variables que caracterizan el daño foliar de los árboles semilleros, no se observan tendencias evidentes que las relacionen con la emergencia de plántulas.

Palabras clave: Araucaria araucana, Semillas.

\section{SUMMARY}

The emergence of seedlings during an operational plant production program, from seeds of 418 progenies of Araucaria araucana, is evaluated. The progenies were classified in 5 ecological-genetic strata and characterized according to the incidence of the disease "foliar damage of the araucaria" on the mother-trees from where the seeds were obtained.

It is observed that the emergence of seedlings of Andean families is slower, but reaches higher values than in coastal families (81.4 and $77.9 \%$ respectively).

So in the different strata separately, as a joint level, the emergence of seedlings had a direct relationship with the size of the seeds.

Regarding to the variables that characterize the foliar damage of the seed trees, no evident tendencies that relate them to the emergence of seedlings were observed.

Keywords: Araucaria araucana, Seeds

\footnotetext{
${ }^{2}$ Instituto Forestal, Sede Bio Bio. bgutierr@infor.cl
} 


\section{INTRODUCCIÓN}

La araucaria (Araucaria araucana (Molina) K. Koch) es una especie emblemática del bosque nativo chileno, sobre la que se realizan prácticas ancestrales asociadas a la alimentación del pueblo pehuenche, está declarada monumento natural, protegida por la convención CITES y constituye un patrimonio nacional que contribuye a la belleza escénica del paisaje y a la protección de las cuencas altas de las regiones de la Araucanía, Bio Bio y los Ríos.

Atendiendo a las amenazas que se ciernen sobre esta especie, particularmente al fenómeno de declinación de origen desconocido denominado como daño foliar de la araucaria (DFA), que se encuentra difundido en toda su área de distribución natural y que prolifera con inusitada rapidez, se ha despertado una gran preocupación que moviliza a distintas entidades, que bajo la coordinación de CONAF buscan desde hace algunos años dar solución a este problema. En este contexto y en el marco del proyecto "Sistema de monitoreo de ecosistemas forestales" (SIMEF), financiado por FAO a través del Fondo Mundial para el Medioambiente (GEF), se decidió cooperar a este esfuerzo desde una nueva óptica, implementando una iniciativa de migración asistida cuyo objetivo es salvaguardar la diversidad genética y el potencial evolutivo de la especie, mediante el establecimiento de bancos de conservación in vivo, estructurados como ensayos de progenies y procedencias fuera del área de distribución natural de la especie. Tales bancos representarán la mayor parte de la diversidad genética de la especie y permitirán obtener material para propagación, mejoramiento, estudios genéticos, sanitarios y otros que contribuyan a la conservación de la especie. Los bancos estarán disponibles para el uso de la comunidad académica y de otras entidades o instituciones vinculadas o interesadas en la conservación de los recursos genéticos forestales.

Para obtener el resultado propuesto, la iniciativa de resguardo genético implementó en el verano-otoño de 2018 una colecta de semillas en las cinco macrozonas (poblaciones o estratos ecológico- genéticos) que conforman el área de distribución natural de araucaria, lo que permitió obtener semillas de un total de 418 árboles madres individuales. La colección de semillas obtenida fue viverizada por personal especializado del Vivero Carlos Douglas de Forestal Mininco SA, para generar las plantas que constituirán el banco de conservación in vivo. En tal contexto, en el presente documento se entrega información compilada durante el proceso de viverización, particularmente la derivada del monitoreo de emergencia de plántulas. Estos antecedentes, ordenados por macrozona, predio y sector de colecta, se presentan en este artículo, donde también se incluye información referente a la relación entre el tamaño de las semillas y la emergencia de plántulas y las eventuales relaciones entre los niveles de afectación por DFA en los árboles madres y la posterior emergencia de plántulas desde sus semillas.

\section{ANTECEDENTES GENERALES DE LA REPRODUCCION DE ARAUCARIA}

Araucaria araucana es una especie dioica, es decir, presenta las estructuras reproductivas masculinas y femeninas en árboles separados, aunque ocasionalmente se presentan individuos monoicos que portan simultáneamente flores masculinas y femeninas; según referencias citadas por Olave (2015) el 1\% de los árboles correspondería a esta última situación.

Los amentos o flores masculinas aparecen entre agosto y septiembre, se ubican en la porción terminal de las ramas y poseen numerosas escamas punzantes, espiraladas, densamente imbricadas que al abrirse liberan abundante polen.

Las flores femeninas, o estróbilos, son conos esféricos 15 a $20 \mathrm{~cm}$ de diámetro, compuestos por numerosas escamas coriáceas y punzantes. Estos conos femeninos se localizan en los extremos de las ramas nuevas y comienzan a desarrollarse hacia finales de noviembre (Marticorena y Rodríguez, 1995; Rodríguez et al., 1983). La polinización es anemófila, ocurre a fines de primavera o principios de verano cuando el viento traslada el polen disponible en nubes compactas desde las flores masculinas a las femeninas. Después de la fertilización el cono 
femenino se lignifica y endurece, para luego abrirse y diseminar las semillas 16 a 18 meses post fertilización (Muñoz, 1984; Marticorena y Rodríguez, 1995).

Cada cono produce entre 120 y 200 semillas, las cuales son relativamente grandes y pesadas (200 a 300 unidades por kilogramo). La cosecha de semillas se realiza en los meses otoñales de marzo y abril, siendo colectada desde el suelo o trepando los árboles (Benítez, 2005).

Donoso (1993) indica que la madurez reproductiva de la especie ocurre aproximadamente a los 25 años, observándose que el máximo de formación de semillas y de floración ocurre con posterioridad a los 40 años.

Debido a su tamaño y peso la diseminación de las semillas se produce básicamente por gravedad, de modo que la mayoría de ellas cae en la vecindad inmediata en torno al árbol que las produjo (Caro, 1995; Muñoz, 1984). No obstante, existen especies animales (loros y ratones) que actúan como vectores que contribuyen a dispersar las semillas, al transportarlas hacia áreas alejadas del árbol madre (Olave, 2015).

Las semillas se caracterizan por perder rápidamente su viabilidad por deshidratación ( 90 a 120 días), por lo mismo se clasifican como semillas recalcitrantes, que no se les puede secar por debajo de $25-40 \%$ de contenido de humedad para almacenarlas por largos periodos Chávez et al. (1999), por cuanto estas se deterioran si su contenido de humedad se reduce demasiado o muy rápidamente (Troncoso, 2015).

Otra característica de las semillas es que presentan latencia fisiológica, no obstante, germinan con relativa facilidad tras el invierno, o al aplicarles un tratamiento pregerminativo de estratificación fría (Benítez, 2005; López et al., 1986; Muñoz, 2010). Debido a esta situación, la siembra se efectúa normalmente durante el periodo otoñal, inmediatamente después de la colecta, emulando así las condiciones naturales de estratificación que se experimentan durante el invierno.

Según Donoso (1978, cit por Zavala, 2018) la germinación de la semilla es semi-hipogea, es decir los cotiledones son visibles, pero permanecen en el suelo; González et al. (2006) la describen como hipogea; otros autores describen la germinación como cryptógea (Burrows et al., 1992; Burrows y Stockey, 1994 cit por Zavala, 2018); por su parte Salazar et al. (2000, cit. por Alvarado y Levet 2014) la describen como hipogea y criptocotilar.

El proceso de germinación se inicia entre 35 a 50 días después de la siembra y finaliza en un lapso de 35 a 45 días (González et al., 2006). El proceso, desde la aparición de la radícula hasta la liberación de los cotiledones, fluctúa entre 15 y 20 días (Urrutia, 1986)

\section{MATERIAL Y MÉTODO}

\section{Colecta y Clasificación de Semillas}

Durante el verano-otoño del año 2018 se implementó una campaña de colecta de semillas de árboles individuales de araucaria en toda el área de distribución natural de la especie. Se colectó semillas de 418 árboles, obteniéndose igual número de lotes familiares que se identificaron, evaluaron y sembraron en forma individual, manteniendo siempre la identificación del árbol madre.

Junto con la obtención de las semillas se registró información del árbol donante, particularmente ubicación, coordenadas y variables descriptivas del nivel de daño foliar exhibido por cada árbol colectado.

Las coordenadas de cada individuo se superpusieron en el plano de definición de estratos ecológicos/genéticos desarrollado por Alarcón (2019) (documento en preparación), lo que 
permitió clasificar los lotes de semillas en los 5 estratos o macrozonas que se muestran en la Figura $N^{\circ} 1$. Estas macrozonas se utilizaron posteriormente para agrupar la información de la emergencia de plántulas de las 418 familias evaluadas.

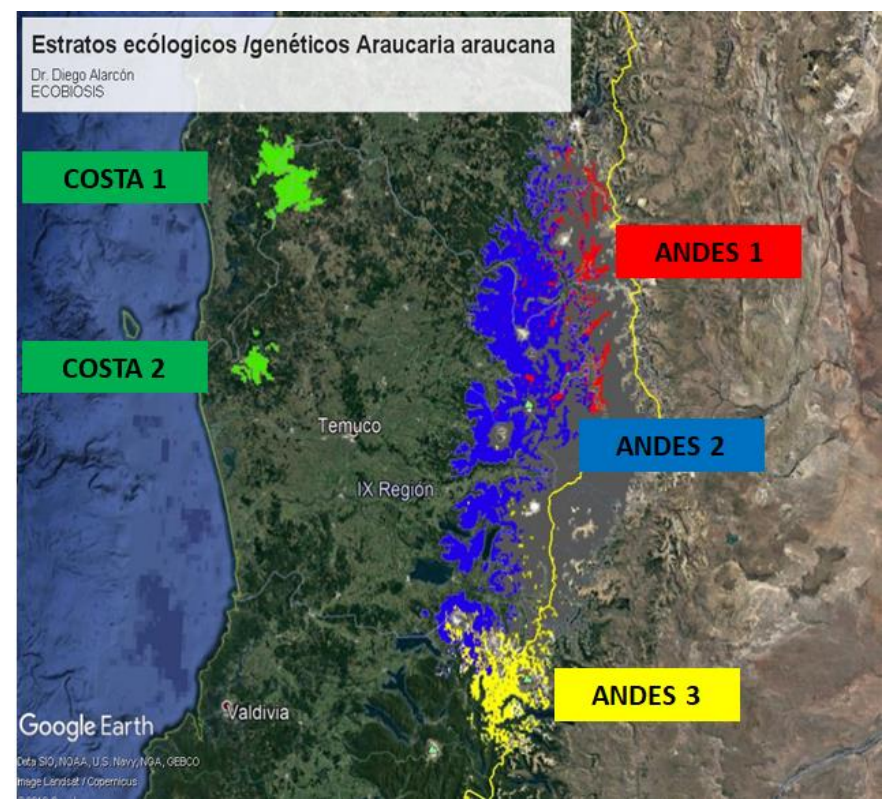

(Fuente: Alarcón, 2019 documento en preparación)

Figura $\mathrm{N}^{\circ} 1$

CLASIFICACIÓN DE LA DISTRIBUCIÓN NATURAL DE Araucaria araucana EN TRES ESTRATOS ECOLÓGICOS/GENÉTICOS

Respecto a la incidencia de daño foliar, cada árbol fue caracterizado en función de las variables y rangos definidos por Reyes et al. (2017), las cuales se sintetizan en el Cuadro $\mathrm{N}^{\circ} 1$. Esta información se utilizó para evaluar la existencia de relaciones entre el daño y la emergencia de plántulas.

\section{Cuadro $N^{\circ} 1$
VARIABLES UTILIZADAS PARA CARACTERIZAR EL DAÑO FOLIAR EXHIBIDO POR LOS ÁRBOLES DESDE DONDE SE OBTUVO LA COLECCIÓN DE SEMILLAS}

\begin{tabular}{|c|l|l|c|}
\cline { 2 - 4 } \multicolumn{1}{c|}{ Categoria } & \multicolumn{1}{c|}{ Variables de Daño } \\
\cline { 2 - 4 } & \multicolumn{1}{c|}{ Localización } & $\begin{array}{c}\text { Proporción de } \\
\text { Copa Afectada } \\
\text { (\%) }\end{array}$ \\
\hline \multirow{4}{*}{ Rangos } & Sano & Sano & 0 \\
\cline { 2 - 4 } & Una rama clorótica & Parte baja de copa & $<25$ \\
\cline { 2 - 4 } & Más de una rama clorótica & Parte media de copa & 25 a 50 \\
\cline { 2 - 4 } & Menos de un tercio sano & Tercio superior de copa & $>75$ \\
\cline { 2 - 4 } & Muerto & Toda la copa & $>75$ \\
\hline
\end{tabular}

(Fuente: Reyes et al., 2017) 
Posteriormente, las semillas de cada familia fueron evaluadas en laboratorio, en términos de tamaño y peso, información que para efectos de este análisis se sintetizó en la variable número de semillas por kilo de semilla limpia. Posteriormente se correlacionó esta variable con la emergencia de plántulas, tanto a nivel de familias individuales como agrupadas en estratos ecológicos-genéticos (macrozonas).

\section{Emergencia de Plántulas y Análisis de Datos}

Una vez colectada, la colección de semillas se mantuvo almacenada en cámara de frío a temperatura de $3^{\circ} \mathrm{C}$ hasta el momento de su siembra, en junio de 2018. La siembra se realizó en invernadero, en dependencias del Vivero Carlos Douglas de Forestal Mininco S.A., usando bandejas contenedoras de plástico con 104 cavidades de 125 cc y corteza de pino compostada como sustrato (Figura $N^{\circ} 2$ ). En función de la cantidad de semillas de cada familia se sembró entre 1 y 2 bandejas para cada una de ellas, registrando el número de semillas sembradas e identificando individualmente las bandejas con el código correspondiente al lote familiar de semillas. El proceso de viverización se efectuó de acuerdo a protocolos y estándares de calidad del vivero.
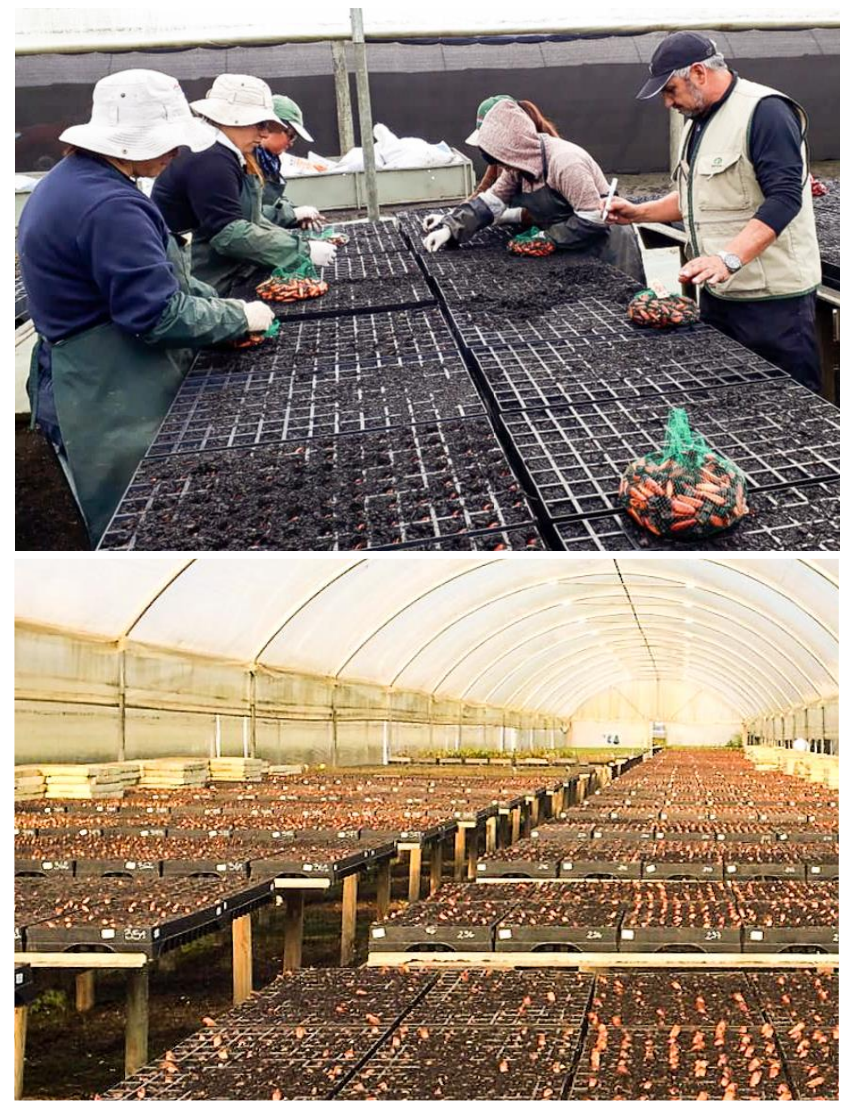

Figura $\mathbf{N}^{\circ} 2$

SIEMBRA DE SEMILLAS (arriba) Y VISTA GENERAL DE LA SIEMBRA (abajo) 
La emergencia de plántulas se monitoreó en dos ocasiones, octubre de 2018 y enero de 2019 , tras 4 y 7 meses transcurridos desde la siembra. En ambas ocasiones se procedió al conteo de la totalidad de las plántulas de cada familia.

Estos datos expresados en porcentaje respecto al número de semillas sembradas por familia, se agruparon a nivel de procedencias y macrozonas para presentarlos en tablas y cuadros; la misma información a nivel de macrozonas se relacionó con parámetros de la semilla, específicamente con su tamaño expresado en número de semillas por kilo, mediante gráficos y coeficientes de correlación línea.

El mismo procedimiento se utilizó para representar las eventuales relaciones entre la emergencia de plántulas y los parámetros descriptivos del daño observado en los árboles desde donde tales semillas fueron obtenidas.

Es necesario aclarar que a diferencia de lo que suele ser habitual, en este artículo se analiza la emergencia de plántulas y no la germinación.

La primera corresponde a una situación más real que permite caracterizar el comportamiento de las semillas durante la viverización, pero constituye una respuesta física de la siembra más que fisiológica del proceso de germinación de las semillas. Ambas evaluaciones tienen aplicaciones y objetivos distintos.

Se debe entender que los resultados de un ensayo de germinación se realizan bajo condiciones ambientales óptimas para que el proceso se produzca y estos se utilizan para precisar requerimientos de pretratamientos en las semillas $y$, por lo tanto, los valores de emergencia de plántulas en vivero o terreno no tienen por qué ser los mismos que entrega el análisis de germinación en laboratorio.

Lo normal es que la emergencia de plántulas sea menor porque, difícilmente, las condiciones ambientales de un vivero son del todo las ideales para que se produzca la germinación (Escobar, 2007).

\section{RESULTADOS Y DISCUSIÓN}

\section{Colecta y Clasificación de Semillas}

Se colectó semilla de un total de 418 árboles, de ellos 47 corresponden a la distribución costa y 371 a la distribución andina (Cuadro $N^{\circ} 2$ ).

En la colección evaluada, el número de semillas por kilogramo, variable que orienta respecto al tamaño de las semillas, alcanza un valor promedio de 266.

Este parámetro fluctúa ampliamente entre familias, las semillas más grandes corresponden a una familia de la macrozona ANDES1 con 144 unidades por kilogramo, mientras que las más pequeñas se encuentran en una familia de COSTA2 con 505 unidades por kilo.

Los valores medios de las familias agrupados a nivel de macrozonas evidencian que las semillas de la zona andina (264 unidades por kilogramo) son de mayor tamaño que las semillas de las procedencias costeras (282 unidades por kilogramo), situación que se expresa gráficamente en la Figura $\mathrm{N}^{\circ} 3$. 


\section{Cuadro $\mathrm{N}^{\circ} 2$}

DETALLE DEL NÚMERO DE FAMILIAS DE QUE COMPONEN LA COLECCIÓN DE SEMILLAS

\begin{tabular}{|c|c|c|c|c|c|}
\hline Macrozona & $\begin{array}{l}\text { Predio } \\
\text { Sector }\end{array}$ & $\begin{array}{l}\text { Familias } \\
\text { Colectadas } \\
\qquad\left(\mathrm{N}^{\circ}\right)\end{array}$ & Macrozona & $\begin{array}{l}\text { Predio } \\
\text { Sector }\end{array}$ & $\begin{array}{c}\text { Familias } \\
\text { Colectadas } \\
\left(\mathrm{N}^{\circ}\right)\end{array}$ \\
\hline \multirow{14}{*}{ COSTA 1} & Nahuelbuta & 21 & \multirow{37}{*}{ ANDES 2} & Pichimalín & 5 \\
\hline & Cam A. P. E.A & 1 & & Lonquimay & 8 \\
\hline & Cam Caramavida-Trongol & 1 & & Cuesta Las Raíces & 8 \\
\hline & Camino a Cerro Anay & 2 & & Marimenuco & 8 \\
\hline & Camino a Piedra del Águila & 3 & & Marimenuco & 8 \\
\hline & Cerro Anay & 1 & & PN Conguillío & 46 \\
\hline & Desvío Cerro Anay & 2 & & Captrén & 9 \\
\hline & Estacionamiento & 2 & & Centro de esquí & 6 \\
\hline & Mirador & 1 & & Conguillío & 16 \\
\hline & Piedra del Águila & 1 & & Conguillío sendero & 2 \\
\hline & Portería Arauco & 3 & & Conguillío-Cabañas & 1 \\
\hline & Portería Malleco & 1 & & Lago -Restaurant & 1 \\
\hline & Primer cruce & 2 & & Las Cabañas & 2 \\
\hline & Sendero Anay & 1 & & Los Paraguas & 4 \\
\hline Subtotal COS & TA 1 & 21 & & Nevados de Vilcún & 2 \\
\hline \multirow{4}{*}{ COSTA 2} & Villa Araucarias & 26 & & Sin Sector & 3 \\
\hline & La Cabaña & 2 & & PN Huerquehue & 32 \\
\hline & Las Mercedes & 1 & & Quinchol & 32 \\
\hline & Pte. El Diablo & 23 & & PN Villarrica & 3 \\
\hline \multicolumn{2}{|c|}{ Subtotal COSTA 2} & 26 & & Chinay Palguín & 2 \\
\hline \multirow{18}{*}{ ANDES 1} & RN Las Nalcas & 35 & & Coñaripe & 1 \\
\hline & Guardería de Conaf & 35 & & RN Las Nalcas & 11 \\
\hline & RN Ralco & 68 & & C/ RN Las Nalcas & 3 \\
\hline & Adecahuin & 15 & & Guardería de Conaf & 8 \\
\hline & Agua de la Argentina & 6 & & RN Malalcahuello & 32 \\
\hline & Caicune & 5 & & Coloradito & 10 \\
\hline & Guardería de Conaf & 3 & & Corralco & 1 \\
\hline & La mula & 8 & & Corralco Guardería & 3 \\
\hline & La vega & 3 & & Corralco Hotel & 14 \\
\hline & La vega c/ La mula & 5 & & El trueno & 2 \\
\hline & La Vizcacha & 1 & & Guardería Corralco & 1 \\
\hline & Los Chenques & 1 & & Hotel Corralco & 1 \\
\hline & Piedra Santa & 4 & & RN Ralco & 29 \\
\hline & Provile & 12 & & Acceso Volcán & 5 \\
\hline & Sendero Laguna & 2 & & Adecahuin & 18 \\
\hline & Sendero Vega & 1 & & La Vega & 4 \\
\hline & SIN GPS & 1 & & Ojo de Agua & 2 \\
\hline & Vega Ralco & 1 & \multicolumn{2}{|c|}{ Subtotal ANDES 2} & 227 \\
\hline \multicolumn{2}{|c|}{ Subtotal ANDES 1} & 103 & \multirow{4}{*}{ ANDES 3} & PN Villarrica & 41 \\
\hline \multirow{8}{*}{ ANDES 2} & Icalma & 38 & & Chianaí-Palguín & 1 \\
\hline & Huillinco & 4 & & Mamuil Malal & 22 \\
\hline & Pudu Mahuida & 3 & & Puesco & 18 \\
\hline & Rincón K Icalma & 28 & \multicolumn{2}{|c|}{ Subtotal ANDES 3} & 41 \\
\hline & Tiltilco & 3 & & & \\
\hline & Cruzaco & 20 & \multicolumn{2}{|c|}{ Subtotal COSTA (1 y 2) } & 47 \\
\hline & \begin{tabular}{|l|} 
Cruzaco \\
\end{tabular} & 10 & \multicolumn{2}{|c|}{ Subtotal ANDES (1, 2 y 3$)$} & 371 \\
\hline & Cumillán & 5 & \multicolumn{2}{|l|}{ TOTAL } & 418 \\
\hline
\end{tabular}




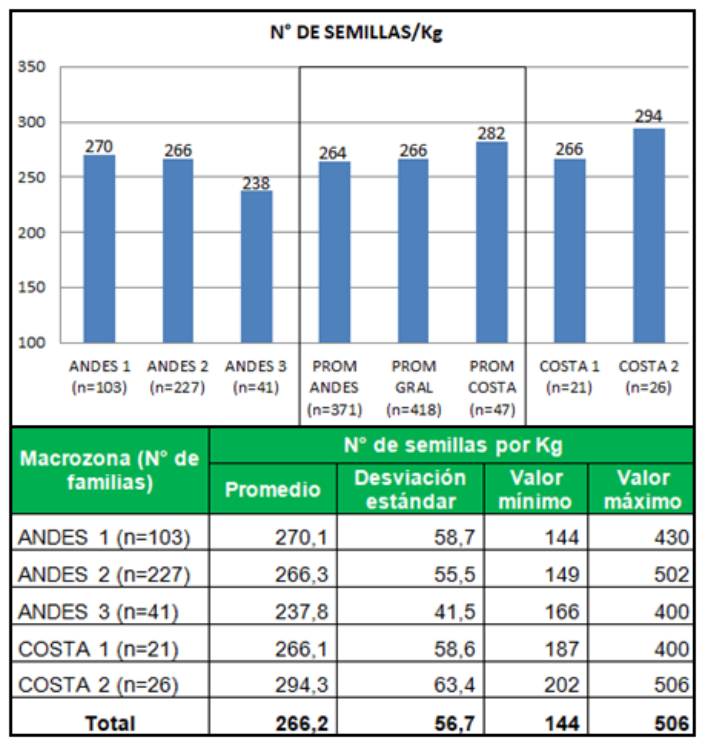

\section{Figura $\mathbf{N}^{\circ} 3$ \\ CARACTERIZACIÓN DEL NÚMERO DE SEMILLAS POR KILOGRAMO DE 418 FAMILIAS AGRUPADAS SEGÚN MACROZONAS}

Los valores obtenidos resultan coincidentes con los registrados en la bibliografía; el número medio de semillas por kilogramo de la colección evaluada es de 266,2 , muy similar a las 260 unidades por kilogramo y 3,8 g/piñón que mencionan como valores medios Muñoz (1984) y Caro (1995). Respecto de la variación observada en el número de semillas por kilogramo, esta también es relativamente coincidente con la documentada en la bibliografía; Zavala (2018), en evaluaciones efectuadas durante tres años consecutivos en la localidad de Lonquimay, señala valores extremos de producción de semillas que fluctúan entre 190 y 523 unidades por kilogramo. El mismo autor menciona valores medios por árbol de 297 a 405 semillas por kilogramo. Por su parte, para el área costera Troncoso (2015) señala valores en el rango de 267 a 348 semillas por kilogramo para la población de Villa Las Araucarias. En ambos casos los valores registrados en zona andina y costera resultan más altos y denotan semillas más pequeñas que las obtenidas en la colección evaluada en este capítulo.

Las variaciones en el tamaño y producción de semillas han tratado de explicarse con distintos argumentos, siendo el más frecuente el que dice relación con la disponibilidad de recursos durante la fase reproductiva, señalándose que la producción de frutos y semillas está condicionada por los niveles de factores limitantes (Zavala, 2018).

En cuanto a las variables que describen la incidencia del daño foliar de la araucaria (categoría de daño, localización del daño y porcentaje de copa afectada) en los árboles desde los cuales se colectó la semilla, se observa una distribución similar para las cinco macrozonas evaluadas (Figura $\mathrm{N}^{\circ} 4$ ); en general los árboles de todas las familias y de todas las macrozonas se concentran en la categoría "más de una rama clorótica", con el daño concentrado principalmente en la parte media y baja de la copa, y con una proporción de copa afectada que fluctúa entre 25 y $50 \%$. De este análisis se desprende también que la enfermedad daño foliar de la araucaria se encuentra ampliamente difundida en toda el área de distribución natural de la especie, y que todos los árboles desde los que se cosechó semillas presentaban algún grado de afectación por esta. 

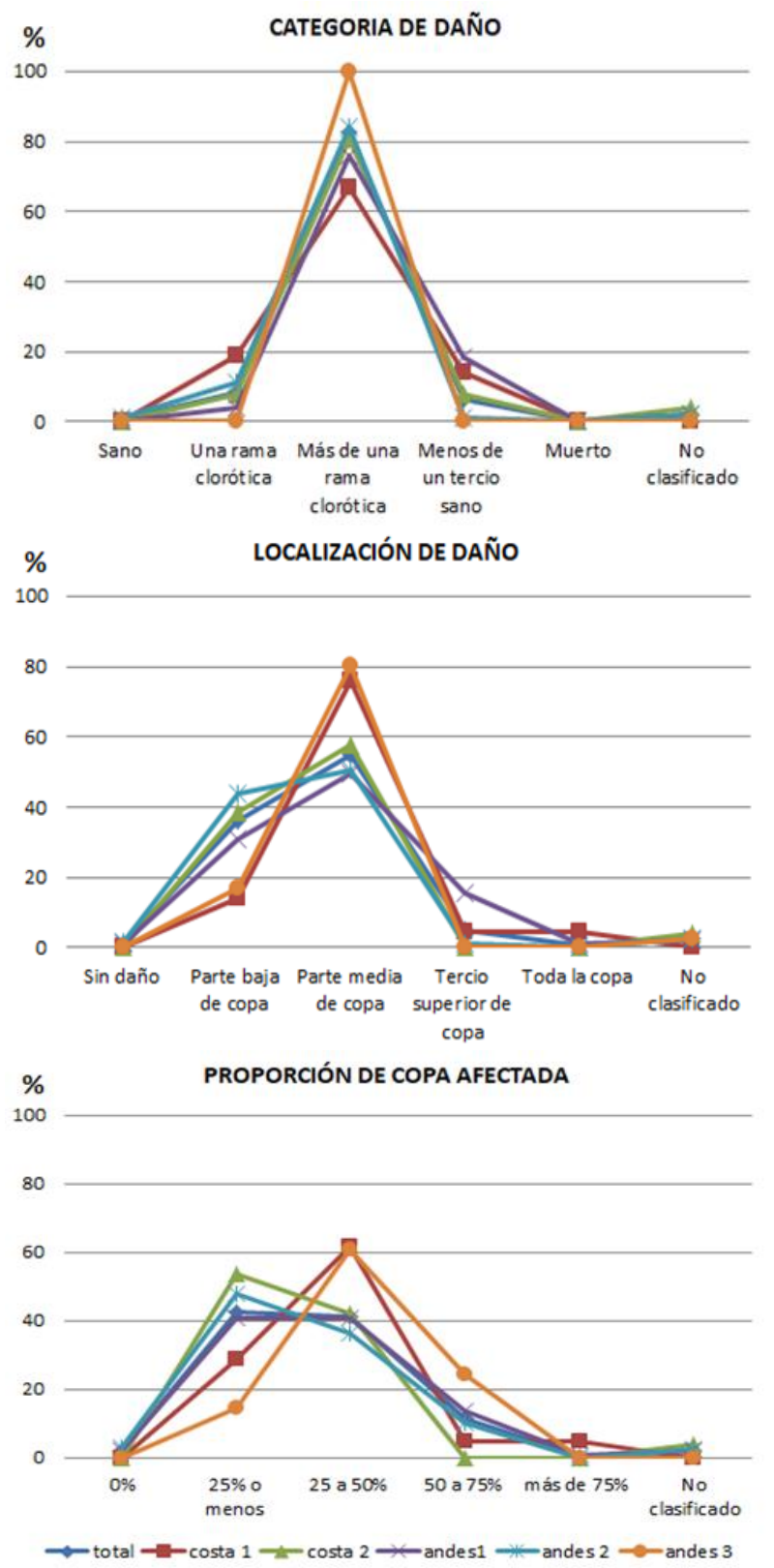

Figura $\mathrm{N}^{\circ} 4$

CARACTERIZACIÓN DE LA INCIDENCIA DE LA ENFERMEDAD DAÑO FOLIAR EN LOS 418 ÁRBOLES DESDE DONDE SE OBTUVO LA COLECCIÓN DE SEMILLAS 


\section{Emergencia de Plántulas}

La primera evaluación de emergencia de plántulas se efectuó en octubre de 2018, cuatro meses después de la siembra, y alcanzó un valor medio de 58,9\% (Figura $\mathrm{N}^{\circ} 5$ ). En la ocasión se observó que en las procedencias costeras la germinación y emergencia de plántulas $(68,1 \%)$ se manifiesta antes que en las procedencias andinas $(57,8 \%)$.

En una segunda evaluación efectuada 7 meses post siembra, en enero de 2019, se constató un aumento general de la emergencia de plántulas, que subió de 58,9 a $81,1 \%$. Este incremento fue de mayor envergadura para las procedencias andinas, las cuales a pesar de exhibir una germinación más lenta alcanzaron valores de emergencia de plántulas superiores a los de las procedencias costeras (Figura $\mathrm{N}^{\circ} 6$; Cuadro $\mathrm{N}^{\circ} 3$ ).

Considerando la evaluación de enero 2019 (7 meses post siembra), en promedio la emergencia de plantas de los 418 lotes de semillas alcanzó a un $81 \%$, siendo mayor en las semillas de la macrozona ANDES $(81,4 \%)$ que en la macrozona COSTA $(77,9 \%)$. En el primer caso el valor corresponde a 371 progenies con semillas que en promedio son algo más grandes que las de la costa $(264 \mathrm{sem} / \mathrm{Kg})$, mientras que en el caso de COSTA corresponde a 47 progenies que en promedio presentan $282 \mathrm{sem} / \mathrm{Kg}$. El detalle de la emergencia de plántulas a nivel de predios y sectores dentro de cada macrozona se presenta en el Cuadro $\mathrm{N}^{\circ} 4$.

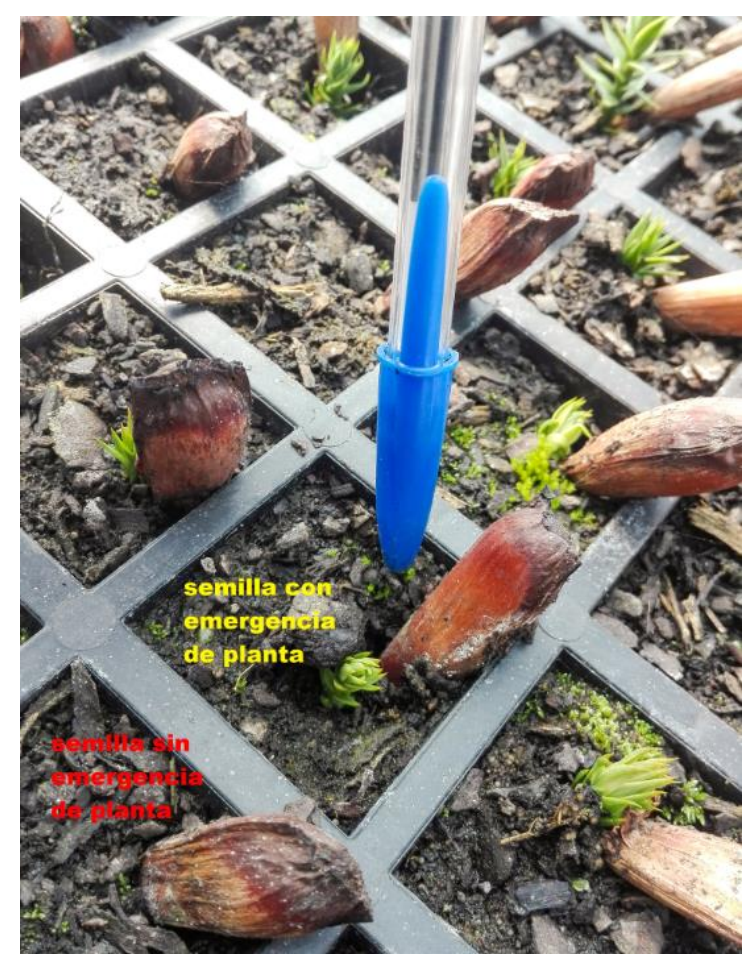

Figura $N^{\circ} 5$

EMERGENCIA DE PLÁNTULAS 


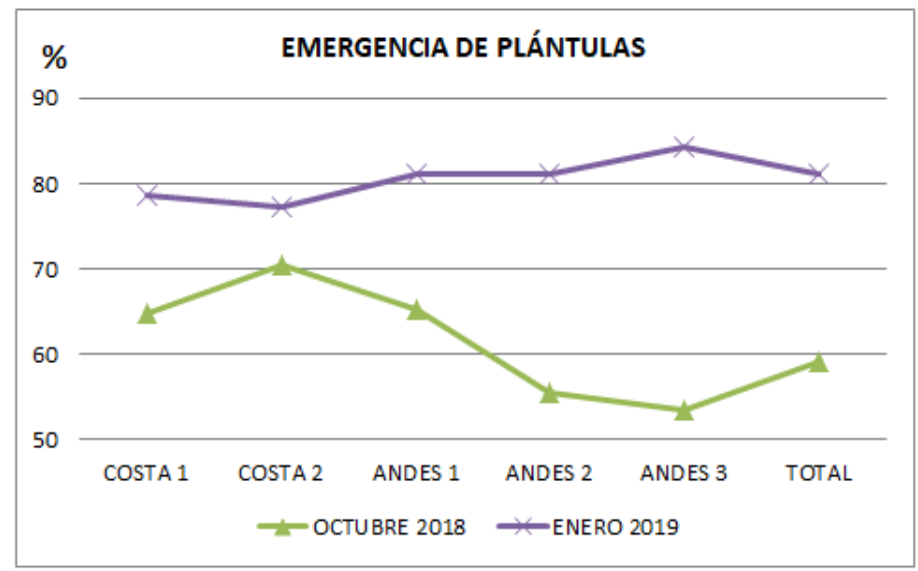

Figura $N^{\circ} 6$

EMERGENCIA PROMEDIO DE PLÁNTULAS DE 418 FAMILIAS AGRUPADAS POR MACROZONAS A LOS 4 Y 7 MESES POST SIEMBRA (SIEMBRA EN JUNIO DE 2018)

Cuadro $\mathrm{N}^{\circ} 3$

ESTADÍSTICA DESCRIPTIVA DE EMERGENCIA DE PLÁNTULAS PARA 418 FAMILIAS SEMBRADAS EN JUNIO 2018

\begin{tabular}{|c|c|c|c|c|c|}
\hline \multirow{2}{*}{ Macrozona } & \multirow{2}{*}{$\begin{array}{l}\text { Familias } \\
\text { Colectadas } \\
\qquad\left(\mathbf{N}^{\circ}\right)\end{array}$} & \multirow{2}{*}{ Parámetro } & \multirow{2}{*}{$\begin{array}{c}\text { Semillas } \\
\text { Sembradas por } \\
\text { Familia } \\
\left(\mathbf{N}^{\circ}\right)\end{array}$} & \multicolumn{2}{|c|}{$\begin{array}{c}\text { Emergencia de Plántulas } \\
(\%)\end{array}$} \\
\hline & & & & Octubre 2018 & Enero 2019 \\
\hline COSTA 1 & 21 & $\begin{array}{l}\text { PROM } \\
\text { RANGO }\end{array}$ & $\begin{array}{c}187 \\
{[54-208]} \\
\end{array}$ & $\begin{array}{c}64,8 \\
{[42,6-81,4]} \\
\end{array}$ & $\begin{array}{c}78,6 \\
{[42,6-93,0]} \\
\end{array}$ \\
\hline COSTA 2 & 26 & $\begin{array}{l}\text { PROM } \\
\text { RANGO }\end{array}$ & $\begin{array}{c}194 \\
{[108-208]} \\
\end{array}$ & $\begin{array}{c}70,5 \\
{[33,0-91,5]} \\
\end{array}$ & $\begin{array}{c}77,2 \\
{[34,6-97,5]} \\
\end{array}$ \\
\hline ANDES 1 & 103 & $\begin{array}{l}\text { PROM } \\
\text { RANGO }\end{array}$ & $\begin{array}{c}202 \\
{[165-312]} \\
\end{array}$ & $\begin{array}{c}65,1 \\
{[22,2-88,9]} \\
\end{array}$ & $\begin{array}{c}81,0 \\
{[30,3-99,0]}\end{array}$ \\
\hline ANDES 2 & 227 & $\begin{array}{l}\text { PROM } \\
\text { RANGO }\end{array}$ & $\begin{array}{c}198 \\
{[78-208]} \\
\end{array}$ & $\begin{array}{c}55,5 \\
{[13,1-89,1]} \\
\end{array}$ & $\begin{array}{c}81,1 \\
{[32,2-98,5]}\end{array}$ \\
\hline ANDES 3 & 41 & $\begin{array}{l}\text { PROM } \\
\text { RANGO }\end{array}$ & $\begin{array}{c}198 \\
{[99-208]}\end{array}$ & $\begin{array}{c}53,5 \\
{[28,6-76,0]} \\
\end{array}$ & $\begin{array}{c}84,2 \\
{[64,1-94,3]}\end{array}$ \\
\hline TOTAL & 418 & $\begin{array}{l}\text { PROM } \\
\text { RANGO }\end{array}$ & $\begin{array}{r}198,2 \\
{[54-312]} \\
\end{array}$ & $\begin{array}{c}59,1 \\
{[13,1-91,5]} \\
\end{array}$ & $\begin{array}{c}81,0 \\
{[30,3-99,0]} \\
\end{array}$ \\
\hline
\end{tabular}




\section{EMERGENCIA DE PLÁNTULAS 418 FAMILIAS 4 Y 7 MESES POST SIEMBRA}

\begin{tabular}{|c|c|c|c|c|c|c|c|}
\hline \multirow{3}{*}{ Macrozona } & \multirow{3}{*}{$\begin{array}{l}\text { Predio } \\
\text { Sector }\end{array}$} & \multirow{3}{*}{$\begin{array}{c}\begin{array}{c}\text { Familias } \\
\text { Colectadas }\end{array} \\
\left(\mathrm{N}^{\circ}\right)\end{array}$} & \multirow{3}{*}{$\begin{array}{c}\begin{array}{c}\text { Semillas } \\
\text { Sembradas }\end{array} \\
\left(\mathrm{N}^{\circ}\right)\end{array}$} & \multicolumn{4}{|c|}{ Emergencia Plántulas } \\
\hline & & & & \multicolumn{2}{|c|}{$\begin{array}{c}4 \text { meses post siembra } \\
\text { (Octubre 2018) }\end{array}$} & \multicolumn{2}{|c|}{$\begin{array}{c}7 \text { meses post siembra } \\
\text { (Enero 2019) }\end{array}$} \\
\hline & & & & $\left(\mathrm{N}^{\circ}\right)$ & $(\%)$ & $\left(\mathrm{N}^{\circ}\right)$ & $(\%)$ \\
\hline \multirow{14}{*}{ COSTA 1} & Nahuelbuta & 21 & 3.929 & 2.569 & 65,4 & 3.108 & 79,1 \\
\hline & Cam A.P. E.A & 1 & 200 & 132 & 66,0 & 174 & 87,0 \\
\hline & Cam Caramavida-Trongol & 1 & 202 & 98 & 48,5 & 129 & 63,9 \\
\hline & Camino a Cerro Anay & 2 & 397 & 290 & 73,0 & 325 & 81,9 \\
\hline & Camino a Piedra del Águila & 3 & 553 & 403 & 72,9 & 466 & 84,3 \\
\hline & Cerro Anay & 1 & 202 & 122 & 60,4 & 183 & 90,6 \\
\hline & Desvío Cerro Anay & 2 & 398 & 225 & 56,5 & 284 & 71,4 \\
\hline & Estacionamiento & 2 & 315 & 199 & 63,2 & 252 & 80,0 \\
\hline & Mirador & 1 & 54 & 23 & 42,6 & 23 & 42,6 \\
\hline & Piedra del Águila & 1 & 208 & 155 & 74,5 & 170 & 81,7 \\
\hline & Portería Arauco & 3 & 599 & 315 & 52,6 & 434 & 72,5 \\
\hline & Portería Malleco & 1 & 200 & 139 & 69,5 & 172 & 86,0 \\
\hline & Primer cruce & 2 & 400 & 307 & 76,8 & 314 & 78,5 \\
\hline & Sendero Anay & 1 & 201 & 161 & 80,1 & 182 & 90,5 \\
\hline \multicolumn{2}{|c|}{ Sub total COSTA 1} & 21 & 3.929 & 2.569 & 65,4 & 3.108 & 79,1 \\
\hline \multirow{4}{*}{ COSTA 2} & Villa Araucarias & 26 & 5.058 & 3.551 & 70,2 & 3.891 & 76,9 \\
\hline & \begin{tabular}{|l|} 
La Cabaña \\
\end{tabular} & 2 & 398 & 238 & 59,8 & 289 & 72,6 \\
\hline & Las Mercedes & 1 & 200 & 142 & 71,0 & 152 & 76,0 \\
\hline & Pte. El Diablo & 23 & 4.460 & 3.171 & 71,1 & 3.450 & 77,4 \\
\hline \multicolumn{2}{|c|}{ Subtotal COSTA2 } & 26 & 5.058 & 3.551 & 70,2 & 3.891 & 76,9 \\
\hline \multicolumn{2}{|c|}{ TOTAL COSTA 1 Y 2} & 47 & 8.987 & 6.120 & 68,1 & 6.999 & 77,9 \\
\hline
\end{tabular}

\begin{tabular}{|c|c|c|c|c|c|c|c|}
\hline \multirow{2}{*}{ ANDES 1} & RN Las Nalcas & 35 & 7.083 & 4.862 & 68,6 & 6.205 & 87,6 \\
\hline & Guardería de Conaf & 35 & 7.083 & 4.862 & 68,6 & 6.205 & 87,6 \\
\hline \multirow{16}{*}{ ANDES 1} & RN Ralco & 68 & 13.743 & 8.717 & 63,4 & 10.678 & 77,7 \\
\hline & \begin{tabular}{|l|} 
Adecahuin \\
\end{tabular} & 15 & 3.054 & 2.040 & 66,8 & 2.521 & 82,5 \\
\hline & Agua de la Argentina & 6 & 1.202 & 498 & 41,4 & 714 & 59,4 \\
\hline & Caicune & 5 & 1.005 & 686 & 68,3 & 870 & 86,6 \\
\hline & Guardería de Conaf & 3 & 608 & 439 & 72,2 & 453 & 74,5 \\
\hline & La mula & 8 & 1.621 & 1.054 & 65,0 & 1.249 & 77,1 \\
\hline & La vega & 3 & 598 & 443 & 74,1 & 544 & 91,0 \\
\hline & La vega c/ La mula & 5 & 1.022 & 715 & 70,0 & 928 & 90,8 \\
\hline & La Vizcacha & 1 & 200 & 63 & 31,5 & 77 & 38,5 \\
\hline & Los Chenques & 1 & 208 & 120 & 57,7 & 133 & 63,9 \\
\hline & Piedra Santa & 4 & 807 & 534 & 66,2 & 599 & 74,2 \\
\hline & Provile & 12 & 2.403 & 1.498 & 62,3 & 1.866 & 77,7 \\
\hline & Sendero Laguna & 2 & 399 & 233 & 58,4 & 267 & 66,9 \\
\hline & Sendero Vega & 1 & 200 & 135 & 67,5 & 170 & 85,0 \\
\hline & SIN GPS & 1 & 208 & 120 & 57,7 & 150 & 72,1 \\
\hline & Vega Ralco & 1 & 208 & 139 & 66,8 & 137 & 65,9 \\
\hline \multicolumn{2}{|c|}{ Subtotal ANDES 1} & 103 & 20.826 & 13.579 & 65,2 & 16.883 & 81,1 \\
\hline
\end{tabular}


(Cuadro N 4 continuación)

\begin{tabular}{|c|c|c|c|c|c|c|c|}
\hline \multirow{5}{*}{ ANDES 2} & Icalma & 38 & 7.520 & 3.618 & 48,1 & 5.714 & 76,0 \\
\hline & Huillinco & 4 & 707 & 382 & 54,0 & 559 & 79,1 \\
\hline & Pudu Mahuida & 3 & 605 & 277 & 45,8 & 467 & 77,2 \\
\hline & Rincon K Icalma & 28 & 5.608 & 2.613 & 46,6 & 4.188 & 74,7 \\
\hline & Tiltilco & 3 & 600 & 346 & 57,7 & 500 & 83,3 \\
\hline \multirow{4}{*}{ ANDES 2} & Cruzaco & 20 & 3.985 & 1.802 & 45,2 & 3.043 & 76,4 \\
\hline & Cruzaco & 10 & 1.967 & 856 & 43,5 & 1.566 & 79,6 \\
\hline & Cumillán & 5 & 1.014 & 548 & 54,0 & 746 & 73,6 \\
\hline & Pichimalín & 5 & 1.004 & 398 & 39,6 & 731 & 72,8 \\
\hline \multirow{2}{*}{ ANDES 2} & Lonquimay & 8 & 1.612 & 1.055 & 65,4 & 1.325 & 82,2 \\
\hline & Cuesta Las Raíces & 8 & 1.612 & 1.055 & 65,4 & 1.325 & 82,2 \\
\hline \multirow{2}{*}{ ANDES 2} & Marimenuco & 8 & 1.607 & 1.039 & 64,7 & 1.343 & 83,6 \\
\hline & \begin{tabular}{|l} 
Marimenuco \\
\end{tabular} & 8 & 1.607 & 1.039 & 64,7 & 1.343 & 83,6 \\
\hline \multirow{11}{*}{ ANDES 2} & PN Conguillío & 46 & 9.018 & 4.671 & 51,8 & 7.717 & 85,6 \\
\hline & Captrén & 9 & 1.801 & 981 & 54,5 & 1.590 & 88,3 \\
\hline & Centro de esquí & 6 & 1.191 & 693 & 58,2 & 1.012 & 85,0 \\
\hline & Conguillío & 16 & 3.022 & 1.436 & 47,5 & 2.570 & 85,0 \\
\hline & Conguillío sendero & 2 & 401 & 228 & 56,9 & 368 & 91,8 \\
\hline & Conguillío-Cabañas & 1 & 201 & 106 & 52,7 & 174 & 86,6 \\
\hline & Lago -Restaurant & 1 & 198 & 144 & 72,7 & 171 & 86,4 \\
\hline & Las Cabañas & 2 & 408 & 119 & 29,2 & 369 & 90,4 \\
\hline & Los Paraguas & 4 & 792 & 459 & 58,0 & 602 & 76,0 \\
\hline & Nevados de Vilcún & 2 & 407 & 200 & 49,1 & 328 & 80,6 \\
\hline & Sin Sector & 3 & 597 & 305 & 51,1 & 533 & 89,3 \\
\hline \multirow{2}{*}{ ANDES 2} & PN Huerquehue & 32 & 6.336 & 3.569 & 56,3 & 5.338 & 84,2 \\
\hline & Quinchol & 32 & 6.336 & 3.569 & 56,3 & 5.338 & 84,2 \\
\hline \multirow{3}{*}{ ANDES 2} & PN Villarrica & 3 & 601 & 305 & 50,7 & 545 & 90,7 \\
\hline & Chinay Palguín & 2 & 399 & 186 & 46,6 & 360 & 90,2 \\
\hline & Coñaripe & 1 & 202 & 119 & 58,9 & 185 & 91,6 \\
\hline \multirow{3}{*}{ ANDES 2} & RN Las Nalcas & 11 & 2.192 & 1.400 & 63,9 & 1.731 & 79,0 \\
\hline & C/ RN Las Nalcas & 3 & 608 & 352 & 57,9 & 486 & 79,9 \\
\hline & Guardería de Conaf & 8 & 1.584 & 1.048 & 66,2 & 1.245 & 78,6 \\
\hline \multirow{8}{*}{ ANDES 2} & RN Malalcahuello & 32 & 6.238 & 3.665 & 58,8 & 4.830 & 77,4 \\
\hline & Coloradito & 10 & 1.959 & 1.296 & 66,2 & 1.549 & 79,1 \\
\hline & Corralco & 1 & 200 & 115 & 57,5 & 142 & 71,0 \\
\hline & Corralco Guardería & 3 & 590 & 254 & 43,1 & 461 & 78,1 \\
\hline & Corralco Hotel & 14 & 2.721 & 1.535 & 56,4 & 2.116 & 77,8 \\
\hline & El trueno & 2 & 377 & 210 & 55,7 & 239 & 63,4 \\
\hline & Guardería Corralco & 1 & 196 & 120 & 61,2 & 157 & 80,1 \\
\hline & Hotel Corralco & 1 & 195 & 135 & 69,2 & 166 & 85,1 \\
\hline \multirow{5}{*}{ ANDES 2} & RN Ralco & 29 & 5.817 & 3.687 & 63,4 & 4.855 & 83,5 \\
\hline & Acceso Volcán & 5 & 1.011 & 502 & 49,7 & 838 & 82,9 \\
\hline & Adecahuin & 18 & 3.618 & 2.398 & 66,3 & 3.019 & 83,4 \\
\hline & La Vega & 4 & 800 & 602 & 75,3 & 654 & 81,8 \\
\hline & Ojo de Agua & 2 & 388 & 185 & 47,7 & 344 & 88,7 \\
\hline \multicolumn{2}{|c|}{ Subtotal ANDES 2} & 227 & 44.926 & 24.811 & 55,2 & 36.441 & 81,1 \\
\hline \multirow{4}{*}{ ANDES 3} & PN Villarrica & 41 & 8.119 & 4.329 & 53,3 & 6.834 & 84,2 \\
\hline & Chianaí-Palguín & 1 & 200 & 95 & 47,5 & 162 & 81,0 \\
\hline & Mamuil Malal & 22 & 4.398 & 2.255 & 51,3 & 3.623 & 82,4 \\
\hline & Puesco & 18 & 3.521 & 1.979 & 56,2 & 3.049 & 86,6 \\
\hline \multicolumn{2}{|c|}{ Subtotal ANDES 3} & 41 & 8.119 & 4.329 & 53,3 & 6.834 & 84,2 \\
\hline \multicolumn{2}{|c|}{ TOTAL ANDES 1, 2 Y 3} & 371 & 73.871 & 42.719 & 57,8 & 60.158 & 81,4 \\
\hline \multicolumn{2}{|c|}{ TOTAL GENERAL } & 418 & 82.858 & 48.839 & 58,9 & 67.157 & 81,1 \\
\hline
\end{tabular}


La emergencia de plántulas observadas es alta y se condice con los mejores valores de capacidad germinativa reportada para la especie en la bibliografía, particularmente para los procesos de viverización con semilla sometida a tratamientos pregerminativos.

En efecto, existen variados antecedentes de germinación de semillas de Araucaria araucana en ensayos de laboratorio, siembra directa en campo y siembra en vivero, los que, si bien son muy variables, coinciden en señalar valores relativamente alto durante la viverización.

Echeverría et al. (2004) menciona valores de $2 \%$ a $33 \%$ en operaciones de siembra directa en terreno y viverización, respectivamente.

Donoso y Cabello (1978) determinaron una capacidad germinativa de 56\%, para semillas proveniente de la provincia de Cautín sin tratamientos pregerminativos.

Muñoz (2010), informa valores de 51 a $89 \%$ para semilla sin tratar y estratificada, respectivamente.

Sanguinetti y Kitzberger (2009, cit. por Duplancic, 2011), obtuvieron una capacidad germinativa de entre 60 y $95 \%$ para siembras directas con exclusión de depredadores vertebrados.

Zavala (2018) citando diversas fuentes menciona valores de germinación de 70 a $82 \%$.

Otros autores citados por Duplancic (2011) señalan germinación en vivero de 70\%, y de hasta $100 \%$ en algunos ensayos de producción de plantas en vivero.

\section{Relación entre Emergencia de Plántulas, Tamaño de Semillas y Nivel de Daño Foliar en Árboles Madres}

A nivel de árboles individuales se observa que los lotes que cuentan con menos semillas por kilogramo (semillas más grandes) tienen una mayor emergencia de plántulas que los lotes con más semillas por kilo (semilla más pequeña).

La misma tendencia general se observa en las macrozonas de Costa y Andes. La correlación entre ambas variables es escasa y ligeramente negativa (Figura $\mathrm{N}^{\circ} 7$ ).

La relación observada es reconocida en el ámbito de la viverización de plantas, sobre este particular Escobar (2007) indica que generalmente las semillas más pequeñas de una muestra tienen menor viabilidad y mayor latencia que las intermedias y más grandes.

La práctica de calibrar la semilla y preferir la más grande para producir plantas suele implementarse en los viveros y para algunas especies, entre ellas eucaliptos, muchos viveristas evitan utilizar en el proceso de siembra las semillas de menor calibre (más pequeñas) las cuales consideran desecho.

En cuanto a la relación entre emergencia de plántulas y variables descriptivas del daño foliar en los árboles fuente de semillas, se observa una tendencia opuesta entre los árboles de las macrozonas Costa y Andes.

En la primera se aprecia una tendencia natural a que los árboles con mayor incidencia de daño exhiban menor emergencia de plántulas, pero esta tendencia se manifiesta en forma opuesta en los árboles de la macrozona Andes (Figura $\mathrm{N}^{\circ} 8$ ). 

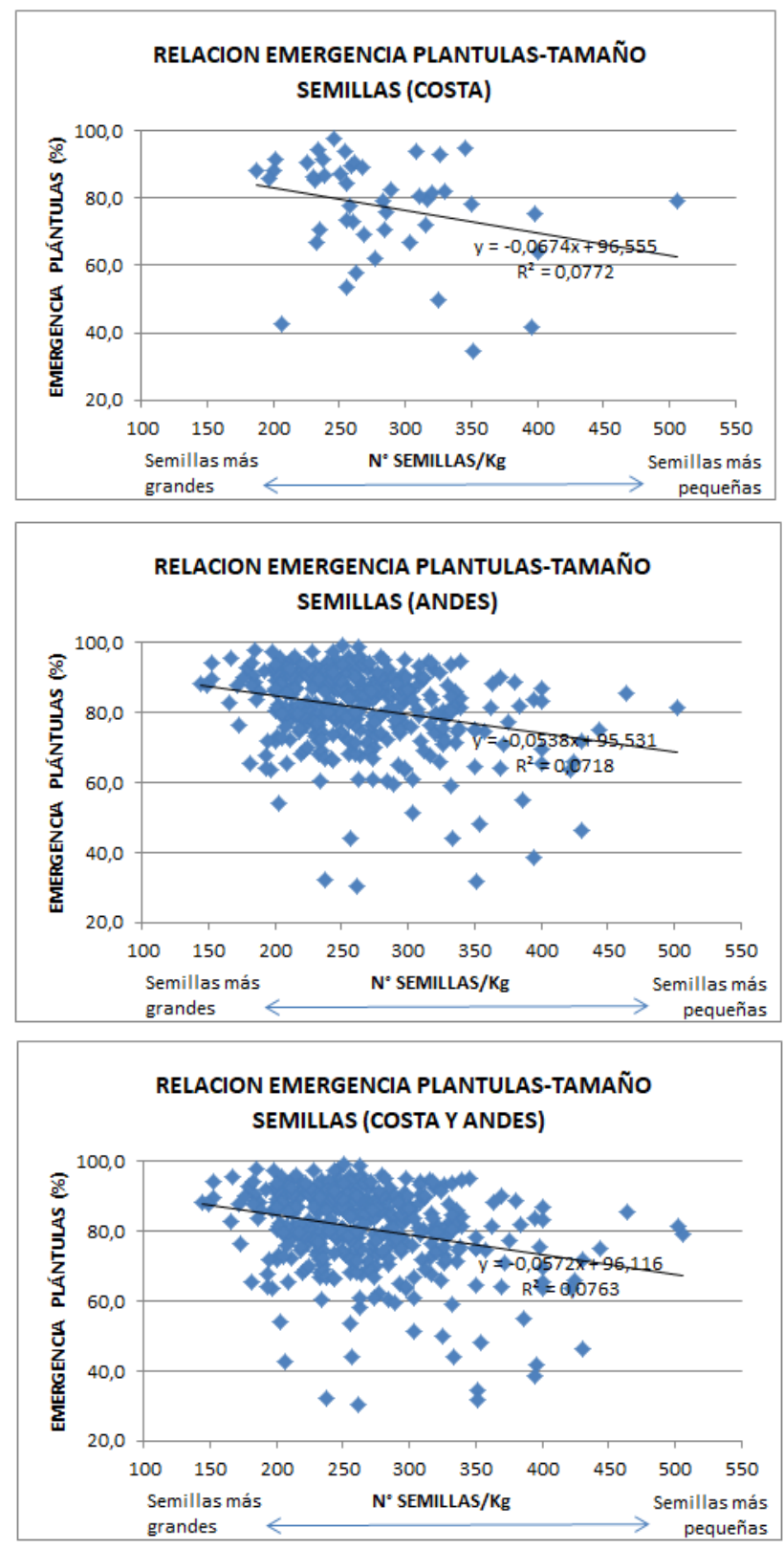

Figura $\mathrm{N}^{\circ} 7$

RELACIÓN ENTRE EMERGENCIA DE PLANTAS Y NÚMERO DE SEMILLAS POR KILOGRAMO 

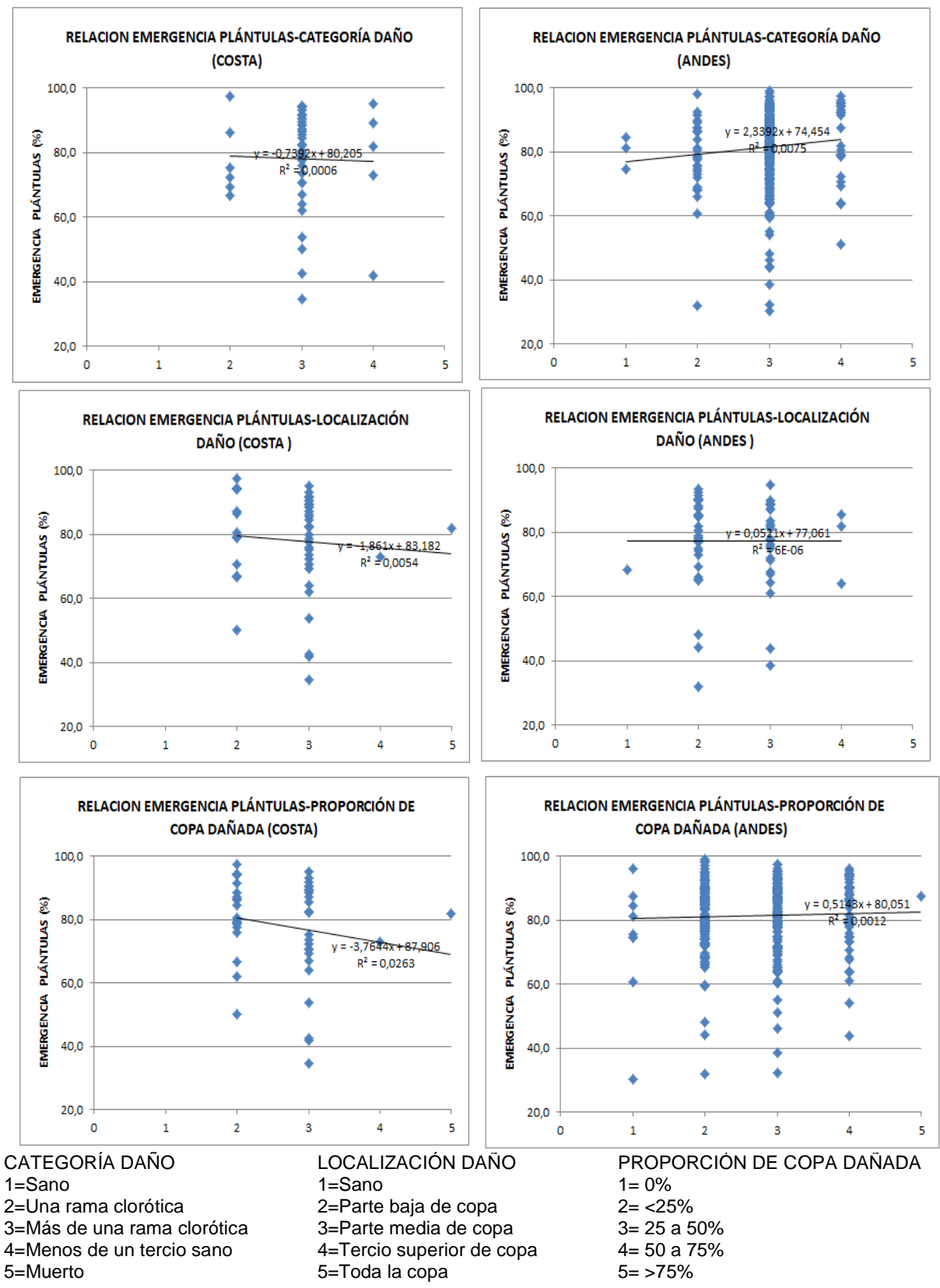

CATEGORIA DAÑO

$1=$ Sano

2=Una rama clorótica

$3=$ Más de una rama clorótica

4=Menos de un tercio sano

$5=$ Muerto
LOCALIZACIÓN DANO

$1=$ Sano

2=Parte baja de copa

$3=$ Parte media de copa

4=Tercio superior de copa

$5=$ Toda la copa 
En la práctica las relaciones observadas parecen ser circunstanciales, observándose gran variación en la emergencia de plántulas dentro de cada valor de las variables que caracterizan al daño (categoría, localización y proporción de copa afectada). Por lo mismo, las tendencias observadas estarían respondiendo más bien al número de árboles en cada nivel y no al nivel propiamente tal.

Análogamente las diferencias entre Costa y Andes obedecerían a esta misma situación, por cuanto entre los árboles de Costa no se registran individuos en el nivel 1 de las variables categoría de daño, localización del daño y proporción de copa afectada; situación que sí ocurre entre los árboles de la macrozona Andes.

\section{CONCLUSIONES}

Existe una natural variación en la emergencia de plantas entre las distintas familias de araucaria. No obstante, los valores son relativamente altos y se condicen con la alta germinación que se reporta para la especie. La emergencia de plántulas, tanto para las semillas procedentes de las macrozonas Costa y Andes, sigue la tendencia general reportada para otras especies en cuanto a que las semillas de mayor tamaño germinan más y producen más plantas que las de tamaño menor.

A nivel de macrozonas, las semillas colectadas en árboles de poblaciones costeras resultaron de tamaño ligeramente menor y exhibieron una emergencia de plántulas más rápida que las semillas de la macrozona Andes. No obstante, los valores de emergencia de plántulas fueron final y definitivamente mayores en las semillas colectadas en poblaciones andinas, las que si bien emergieron más tarde experimentaron incrementos de emergencia en el tiempo mayores que los exhibidos en la macrozona Costa.

En cuanto a la incidencia del daño foliar de los árboles donantes de las semillas, no parece que este sea un factor que incida en la emergencia de plántulas. Al respecto debe tenerse en cuanta, que al momento de la colecta se privilegió a los que presentaban menores niveles de daño.

\section{REFERENCIAS}

Alvarado, A. y Levet, O., 2014. Manual de protocolos de producción de especies utilizadas por el programa de arborización. Corporación Nacional Forestal, Gerencia Forestal, Dpto. de Arborización. Santiago, Chile. 177 p.

Benítez, C., 2005. Viabilidad de las semillas y crecimiento inicial de plántulas de Araucaria araucana (Mol.). K. Koch de la Cordillera de Nahuelbuta en la IX región de Chile. Tesis, Facultad de Ciencias Agropecuarias y Forestales, Universidad Católica de Temuco. Temuco, Chile. 107 p.

Caro, M., 1995. Producción y dispersión de semillas de Araucaria araucana (Mol.) K. Koch, en Lonquimay. Memoria de título de Ing. Forestal. Universidad de Chile. Facultad de Ciencias Agraria y Forestales. Santiago. Chile. 25-51.

Chávez, A.; Mugridge, A.; Fassola, H.; Alegranza, D. y Fernandez, R., 1999. Conservación refrigerada de semillas de Araucaria angustifolia (Bert.) O. Kuntze. Bosque 20(2): 117-124.

Donoso, C. y Cabello, A., 1978. Antecedentes fenológicos y de germinación de especies leñosas chilenas. Departamento de silvicultura. Facultad de Ciencias Forestales. Universidad de Chile. 1: 31-41.

Donoso, C., 1993. Bosques Templados de Chile y Argentina. Variación, estructura y dinámica. Primera Edición. Santiago, Chile. Editorial Universitaria. 484 p.

Duplancic, M., 2011. Germinación de semillas de Araucaria araucana provenientes del bosque xérico norpatagónico. Multequina 20: 113-115. ISSN 1852-7329 on-line 
Echeverría, C.; Zamorano, C. y Cortés, M., 2004. Conservation and restoration of monkey puzzle (Araucaria araucana) forest in Chile. Global Trees Campaign. Final Report. www.globaltrees.org/downloads/ChileReportS.pdf.

Escobar, R., 2007. Manual de viverización. Instituto Forestal, Centro Tecnológico de la Planta Forestal. Concepción, Chile. 230 p.

González, M.; Cortés, M.; Izquierdo, F.; Gallo, L.; Echeverría, C.; Bekkesy, S. y Montaldo, P., 2006. Araucaria araucana (Molina) K. Koch. In Cuneo M eds. Las especies arbóreas de los bosques templados de Chile y Argentina. Valdivia, Chile. Marisa Cuneo Ediciones. p. 36-53.

López, H., Jiménez, G. y Reyes, B., 1986. Algunos antecedentes sobre cosecha, procesamiento y viverización de varias especies nativas. Revista Chile forestal. Documento técnico №5.

Marticorena, C. y Rodríguez, R., 1995. Flora de Chile. Volumen I, Pterydophyta-Gymnospermae. Universidad de Concepción. Concepción, Chile. Editorial Aníbal Pinto. 352pp.

Muñoz, R., 1984. Análisis de la productividad de semillas de Araucaria araucana (Mol.) K. Koch en el área de Lonquimay, IX Región. Tesis. Fac. Cs. Agrarias, Veterinarias y Forestales. Universidad de Chile. Santiago. Chile. $84 \mathrm{p}$.

Muñoz, F., 2010. Evaluación del almacenamiento, germinación de semillas y producción de plantas de Araucaria araucana (Mol.) K. Koch, procedentes de la comuna de Lonquimay, IX Región. Memoria para optar al Título Profesional de Ingeniero Forestal. Universidad de Chile. Santiago, Chile. 49 p.

Olave, F., 2015. Efectos de la aplicación de cortas selectivas en bosques de Araucaria araucana (Mol.) K. Koch ubicados en la región de la Araucanía, Chile. Tesis Doctoral presentada en satisfacción de los requisitos necesarios para optar al grado de Doctor por la Universidad Internacional de Andalucía. Programa de Doctorado compartido Universidad de Andalucía- Universidad de Córdoba. España. 202 p.

Reyes, M.; Pinto, R. y Cruz, P., 2017. Estudios de prospección fitosanitaria en los bosques de Araucaria araucana, para determinar los niveles de afectación de daño foliar en los bosques de las regiones del Biobío, Araucanía y Los Ríos. UFRO-OTERRA. Presentación en seminario internacional International Meeting of the Araucaria. Pucón, Chile. 6-9 noviembre, 2017.

Rodríguez, R.; Matthei, O. y Quezada, M., 1983. Flora Arbórea de Chile. Concepción, Chile. Editorial de la Universidad de Concepción.

Troncoso, A., 2015. Evaluación del proceso de germinación de la especie Araucaria araucana (Mol.) Koch. proveniente del sitio prioritario de conservación de Villa Las Araucarias, Región de La Araucanía. Trabajo de Titulación presentado como parte de los requisitos para optar al Título de Ingeniero en Conservación de Recursos Naturales. Universidad Austral de Chile, Facultad de Ciencias Forestales y Recursos Naturales. Valdivia, Chile. $46 p$.

Urrutia, J., 1986. Análisis bibliográfico y pictórico de semillas y sus procesos germinativos para 32 especies forestales nativas. Tesis, Facultad de Ciencias Forestales, Universidad Austral de Chile. Valdivia, Chile. 64pp.

Zavala, J. 2018. Análisis de la producción, germinación y viabilidad de semillas de Araucaria araucana (Molina) k. Koch, para tres años de fructificación y tres localidades de la comuna de Lonquimay, región de la Araucanía. Memoria para optar a título de Ingeniero Forestal. Universidad de Chile, facultad de Ciencias forestales y de Conservación de la naturaleza. Santiago, Chile. 29 p. 